\title{
Extraction of Document Descriptive Terms with a Linguistic-Based Machine Learning Approach ${ }^{\star}$
}

\author{
Javier Fernández, Elena Montañés, Irene Díaz, José Ranilla, and \\ Elías F. Combarro** \\ Artificial Intelligence Center - University of Oviedo (Spain) \\ ir@aic.uniovi.es
}

\begin{abstract}
In this paper we present a method for extracting relevant words from a document taking into account linguistic information. Form a set of words manually labelled as relevant or not and with the aid of a Machine Learning algorithm, we build a classifier which is able to decide which words from a unseen document must be regarded as relevant. This system is compared with some classical methods (based just on statistical information).
\end{abstract}

\section{Introduction and Previous Work}

One of the main processes in Information Retrieval (IR) and Text Categorisation (TC) is that of transforming documents into a form suitable for automatic processing. The most used representation consists of identifying each document with a vector whose components measure the importance of the different words. This representation is known as bag of words (see [1]). It is interesting to study methods for the removal of non-informative features. This paper explores the use of linguistic knowledge in this process.

A widely adopted approach to feature reduction consists of ordering the words according to a measure of their relevance and selecting those with the highest score. For instance, one can count the total number of appearances $(t f)$ of each word in the corpus and then keep only the most frequent words. The dispersion of the word in the corpus can also be considered with tfxidf defined by $t f x i d f=t f \log \left(\frac{N}{d f}\right)$ where $N$ is the total number of documents and $d f$ is the number of different documents in which the word appears. If the corpus is a set of categorised documents the distribution of a word over the different categories can be considered. This is the case of information gain (IG) [2]

All these approaches have in common the use of mainly statistical information. However, including the syntactic and grammatical function of the words and the location in which they appear, may help to successfully determine the importance of the words in the documents. Some authors 34 do use some lexical information, but usually only a quite restricted one.

* This research has been supported under MCyT and Feder grant TIC2001-3579.

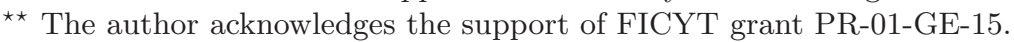




\section{The System and the Experiments}

Our system builds a binary classifier to decide whether a word can be regarded as a descriptive term for a certain document or not. This inductive process starts from a set of attributes of a set of words that have been previously classified either as descriptive or not. In this work we use two Machine Learning (ML) systems to perform this task: Arni-rules [5] and C4.5-rules [6].

Each word is represented by a set of attributes which provide linguistic information of such word in the context of each document under study. Linguistic information is divided into POS-tagging information, representing the lexical functions that a word can play in a document (obtained with Eric Brill's tagger [7]); and term location, including information about the position of the word in the document (the word appears in the title, at the beginning of the document,...). The values of these attributes can be boolean or numerical. The influence of word frequency $(t f)$ is also taken into account.

The attributes of a word in the different documents need to be aggregated. We have adopted two different methods to perform this task. The first one consists of adding the values of the attributes. We will call $t f$-agg this type of aggregation. The second one involves performing the $t f$-agg and then multiplying it by $\log \left(\frac{N}{d f}\right)$. This kind of aggregation will be called tfxidf-agg.

The experiments have been conducted with two corpora: Reuters-21578, which contains short news related to economy published by Reuters during 1987; and Ohsumed.91, which covers all references from 270 medical journals of 1991. We have used all the documents of the categories Alum and Cocoa from Reuters21578 and 60 documents of the category C06 from Ohsumed.91.

\section{Results}

To quantify the performance we use precision and recall. Precision $(P)$ is the percentage of words classified as descriptive which really are, and recall $(R)$ is the percentage of descriptive terms recognized by the system. They are combined by means of $F_{1}$ (cf. [8]) defined by $F_{1}=\frac{1}{\frac{1}{2 P}+\frac{1}{2 R}}$. The experiments are performed using stratified cross validation with 10 folds and 5 repetitions.

In Tables 1 , 2 and 3 we present the averaged $F_{1}$ obtained in the experiments. Results both without stemming (WS) and with stemming (S) according to Porter [9] are reported. We denote POS-tagging attributes by "L", boolean term location attributes by "B" and numeric term location attributes by "N".

The general trends in the three categories and with both systems are similar. First of all, it is clear, with few exceptions, that the use of stemming results in an improvement. On the other hand, the tfxidf-agg shows, in general, better results than the $t f$ one. The combination of POS-tagging and term location seems to improve the performance in many cases, while the inclusion of $t f$ has little impact (in many cases the result is exactly the same). In addition, although the results of Cocoa, Alum and C06 follow the same trend, the $F_{1}$ of the first two is remarkably lower, possibly due to an overfitting problem. 
Table 1. Resulting $F_{1}($ in $\%$ ) in Alum

\begin{tabular}{|c||cc|cc||cc|cc|}
\hline \multicolumn{1}{|c||}{} & \multicolumn{4}{c||}{ Arni-rules } & \multicolumn{3}{c|}{ C4.5-rules } \\
Attributes & \multicolumn{2}{|c|}{$t f$-agg. } & tfxidf-agg. & \multicolumn{3}{|c|}{$t f$-agg. } & \multicolumn{1}{c|}{$t$-aidf-agg. } \\
& WS & S & WS & S & WS & S & WS & S \\
\hline L & 40.16 & 42.40 & 43.40 & 44.83 & 44.87 & 34.27 & 43.83 & 50.34 \\
L+Tf & 40.16 & 45.54 & 43.40 & 47.51 & 44.87 & 32.49 & 43.83 & 49.54 \\
L+B & 34.72 & 41.33 & 35.33 & 39.94 & 41.22 & 46.01 & 38.36 & 51.23 \\
L+B+Tf & 34.72 & 42.65 & 35.33 & 40.87 & 41.61 & 44.29 & 39.19 & 49.85 \\
L+N & 39.20 & 42.74 & 40.55 & 38.70 & 39.85 & 40.82 & 37.10 & 45.94 \\
L+N+Tf & 39.20 & 43.54 & 40.55 & 40.08 & 39.78 & 38.91 & 37.11 & 44.99 \\
\hline
\end{tabular}

Table 2. Resulting $F_{1}$ (in \%) in Cocoa

\begin{tabular}{|c||cc|cc||cc|cc|}
\hline \multicolumn{1}{|c||}{ Attributes } & \multicolumn{4}{c||}{ Arni-rules } & \multicolumn{3}{c|}{ C4.5-rules } \\
& \multicolumn{2}{|c|}{$t f$-agg. } & \multicolumn{2}{c|}{ tfxidf-agg. } & \multicolumn{2}{|c|}{$t f$-agg. } & \multicolumn{2}{c|}{ tfxidf-agg. } \\
& WS & S & WS & S & WS & S & WS & S \\
\hline L & 16.67 & 23.82 & 18.00 & 21.14 & 13.10 & 15.33 & 24.94 & 19.17 \\
L+Tf & 13.79 & 23.11 & 14.97 & 22.75 & 13.31 & 16.40 & 24.46 & 16.20 \\
L+B & 20.93 & 23.36 & 27.74 & 27.46 & 26.42 & 27.86 & 30.14 & 28.57 \\
L+B+Tf & 20.69 & 22.48 & 26.15 & 28.39 & 26.27 & 19.07 & 28.72 & 27.27 \\
L+N & 21.32 & 27.60 & 25.25 & 26.98 & 11.63 & 23.81 & 22.42 & 22.38 \\
L+N+Tf & 21.88 & 26.65 & 25.71 & 26.60 & 12.77 & 17.96 & 21.92 & 19.81 \\
\hline
\end{tabular}

The proposed method is compared with 3 measures of word relevance: $t f$, tfxidf and $I G$. Using them, we rank the words and calculate the resulting $F_{1}$ when the words with highest values are considered as descriptive terms. The results are presented in Table 4, where the filtering level indicates the percentage of words that are supposed to be non-informative. The filtering level obtained with our system ranges from $75 \%$ to $90 \%$ in Alum, from $90 \%$ to $98 \%$ in Cocoa and from $70 \%$ to $88 \%$ in C06, so only the corresponding levels are presented.

If we use both POS-tagging and term location data, stemming is performed, the aggregation is tfxidf-agg and the ML system is C4.5-rules, then the performance of the system is better than that of the traditional measures at all the comparable filtering levels. Namely, we obtain $F_{1}=51.31 \%$ (filtering level of $75.39 \%$ ) in Alum, $F_{1}=28.57 \%$ (filtering level of $93.01 \%$ ) in Cocoa and $F_{1}=56.97 \%$ (filtering level of $77.47 \%$ ) in C06. For other choices of parameters the results are also better than those of the traditional measures for most filtering levels.

\section{Concluding Remarks and Future Work}

We have presented a system for the extraction of informative words from a document which takes into account POS-tagging, term location and statistical information. When comparing the results obtained by traditional filtering measures with those got with a good selection of parameters in our system we find that our method performs better. Additionally, with this system it is not necessary to select the filtering level since it is automatically obtained.

However, some overfitting problems must be solved. Hence it is necessary to study which is the most appropriate number of examples. As an extension of this 
Table 3. Resulting $F_{1}($ in $\%)$ in $\mathrm{C} 06$

\begin{tabular}{|c||cc|cc||cc|cc|}
\hline \multicolumn{1}{|c||}{ Attributes } & \multicolumn{3}{c||}{$t f$-agg. } & \multicolumn{3}{c|}{$t$ txidf-agg. } & \multicolumn{3}{c|}{$t f$-agg. } & \multicolumn{3}{c|}{$t$ C4xidf-agg-rules } \\
& & \multicolumn{3}{c|}{ Arni-rules } & \multicolumn{3}{c|}{} \\
& WS & S & WS & S & WS & S & WS & S \\
\hline L & 48.57 & 48.05 & 41.97 & 54.35 & 34.64 & 47.83 & 51.65 & 59.96 \\
L+Tf & 48.81 & 48.91 & 42.31 & 53.99 & 34.64 & 51.38 & 51.62 & 59.22 \\
L+B & 48.57 & 53.46 & 50.10 & 54.57 & 49.27 & 55.47 & 49.45 & 56.97 \\
L+B+Tf & 48.57 & 53.60 & 49.99 & 55.01 & 49.02 & 54.29 & 49.84 & 57.49 \\
L+N & 54.08 & 58.32 & 53.60 & 59.30 & 57.14 & 60.57 & 57.34 & 62.19 \\
L+N+Tf & 54.60 & 58.73 & 54.16 & 58.76 & 57.14 & 61.14 & 57.24 & 62.62 \\
\hline
\end{tabular}

Table 4. $F_{1}$ (in \%) of $t f, t f x i d f$ and $I G$

\begin{tabular}{|l|c|c|c|c|}
\hline Category & Filtering Level & tf & tfxidf & IG \\
\hline \hline Alum & $75 \%$ & 41.28 & 44.04 & 32.99 \\
& $80 \%$ & 39.29 & 39.29 & 32.65 \\
& $85 \%$ & 35.53 & 36.68 & 32.66 \\
& $90 \%$ & 33.44 & 33.44 & 28.20 \\
\hline \hline Cocoa & $90 \%$ & 22.29 & 24.80 & 19.10 \\
& $95 \%$ & 21.20 & 20.60 & 16.00 \\
\hline \hline C06 & $70 \%$ & 40.21 & 53.12 & 33.80 \\
& $75 \%$ & 45.78 & 48.19 & 30.45 \\
& $80 \%$ & 39.86 & 44.90 & 27.85 \\
& $85 \%$ & 32.98 & 39.63 & 24.20 \\
& $90 \%$ & 25.93 & 31.89 & 18.78 \\
\hline
\end{tabular}

work, we are also interested in studying the impact of considering syntactical information of the words. We would also like to check whether extracting words with the system proposed improves the overall performance of TC.

\section{References}

1. Salton, G., McGill, M.J.: An introduction to modern information retrieval. McGrawHill (1983)

2. Yang, T., Pedersen, J.P.: A comparative study on feature selection in text categorisation. In: Proceedings of ICML'97, 14th International Conference on Machine Learning. (1997) 412-420

3. Barker, K., Cornacchia, N.: Using noun phrase heads to extract document keyphrases. Lecture Notes in Computer Science 1822 (2000) 40-52

4. Turney, P.D.: Learning algorithms for keyphrase extraction. Information Retrieval 2 (2000) 303-336

5. Ranilla, J., Bahamonde, A.: Fan: Finding accurate inductions. International Journal of Human Computer Studies 56 (2002) 445-474

6. Quinlan, J.R.: Constructing decision tree in c4.5. In: Programs of Machine Learning. Morgan Kaufman (1993) 17-26

7. Brill, E.: A Corpus-Based Approach to Language Learning. PhD thesis, Philadelpha, PA (1993)

8. Sebastiani, F.: Machine learning in automated text categorisation. ACM Computing Survey 34 (2002)

9. Porter, M.F.: An algorithm for suffix stripping. Program (Automated Library and Information Systems) 14 (1980) 130-137 